\title{
Development of Indonesia's National Digital Library Network
}

\author{
Ismail Fahmi \\ ismail@itb.ac.id \\ Knowledge Management Research Group \\ Institut Teknologi Bandung \\ Indonesia
}

\section{Introduction}

It has become evident that budgets for libraries in Indonesia are very small. According to The Indonesian University Libraries Forum (FPPTI), every university in Indonesia should allocate $5 \%$ of its budgets for libraries. A survey by this forum in 2003 has shown that only 5 of 125 universities have allocated $5 \%$ of their budgets while $40 \%$ of them allocated less than $2 \%$. With this condition, it is very difficult for the libraries to achieve the standard set by the Ministry of National Education that they should provide minimum of 2 book titles for every course and cover 10\% of their students (Media Indonesia, 2003).

Apparently, the electronic journal subscriptions are also hardly found at most university libraries in this country. In 1999, the Indonesian Cyber-library Society (ICS) has submitted a proposal to the libraries of several big universities for a consortium-based electronic journals subscription ${ }^{1}$. The limitation of budgets and Internet bandwidth made the institutions didn't consider it as their priority. Finally, the existing local resources such as undergraduate final projects, thesis, dissertations, journals, etc. become important sources for students.

Realizing this limitation, 40 people from 23 institutions conducted a meeting in Bandung on October 2000 (Fahmi, et al., 2000). Their main goal was to develop an innovative and collaborative strategy to acquire, maintain, and share local resources. As the ultimate result, they initiated a digital library network, which then is called as the Indonesia Digital Library Network (IndonesiaDLN), and developed an open-source software called Genasha Digital Library $\left(\mathrm{GDL}^{2}\right)$. It was expected that by sharing the local resources as mentioned above, students from an institution will be able to improve their information literacy by accessing resources from other institutions.

IndonesiaDLN can be seen as a network that integrates digital libraries from many institutions in Indonesia by collecting and sharing their local resources in the form of

\footnotetext{
${ }^{1}$ This initiative was raised up during a long discussion in the ICS mailing list in 1999 . The community has no formal organization, but has become the biggest virtual library community in Indonesia.

${ }^{2}$ GDL website: http://gdl.itb.ac.id.
} 
metadata and full texts. Through every digital library server, a user can browse, search, and download any resources shared by the IndonesiaDLN members.

The growth of this network is significantly fast. Only in two years since the first time the network was launched in 2001, the number of new digital libraries joined the IndonesiaDLN has reached 45 members. They are located at the big five islands, namely Java, Sumatra, Kalimantan, Sulawesi, and Irian Jaya as shown by Figure 1. Majorities are from university and also from NGO, government, and individual.

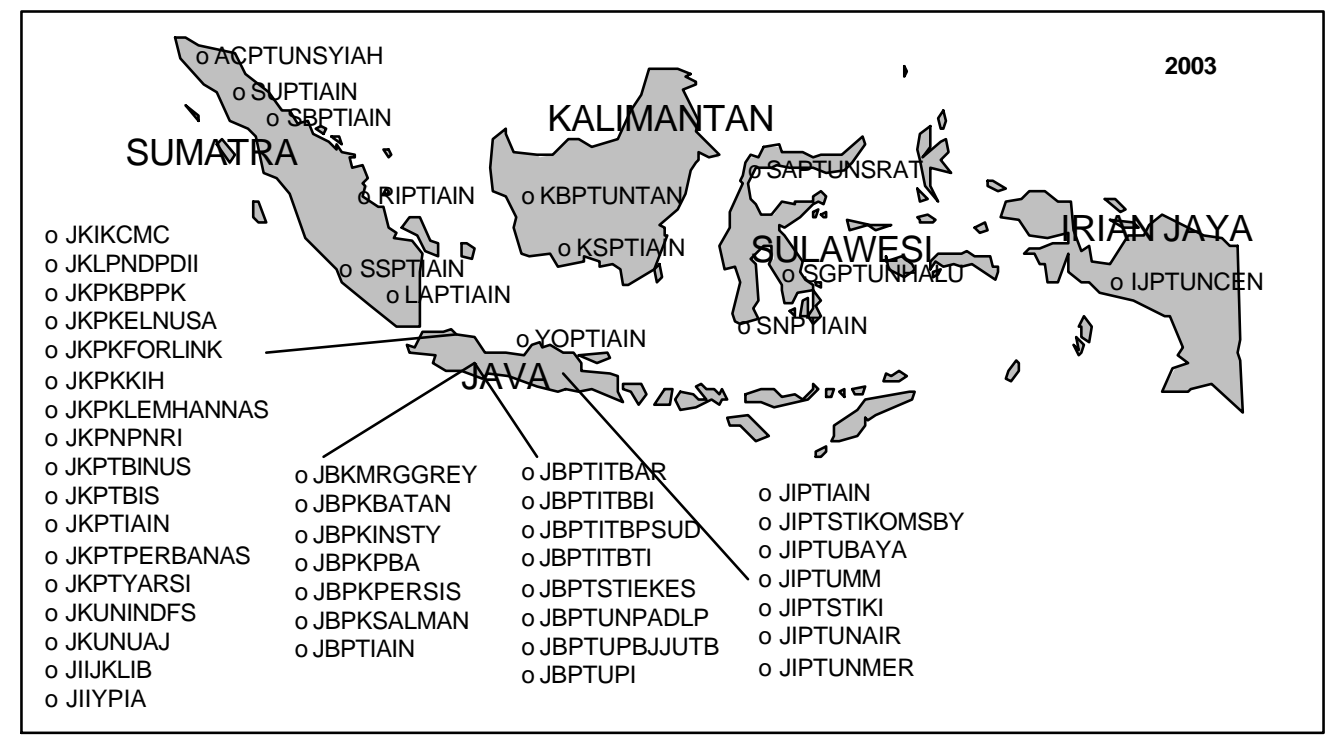

Figure 1. The map of Indonesia islands that denotes the location of the IndonesiaDLN members. Each member is indicated by its PublisherID, such as ACPTUNSYIAH that stands for: AC as Aceh (name of the city), PT as Perguruan Tinggi 'University' (type of the institution) and UNSYIAH as University of Syah Kuala (name of the institution).

This initiative is then followed by other initiatives, which developed regional-based or community-based digital library networks. For example, the Environmental Digital Library Network $\left(\mathrm{JPLH}^{3}\right)$ and Muhammadiyah Digital Library Network (MDLN) are both community-based digital library networks, which were established in 2003; and Public University Link System of East Java (PULSE ${ }^{4}$ ) is a regional-based initiative of 8 universities in East Java, which extends its goals to the delivery of educational material among its members.

This chapter will describe the technical aspect of the IndonesiaDLN, including the basic concept of the network, metadata standards, metadata exchange protocol with our novel approach, and implementation. The statistics of its usage, problems, future works, and conclusion also will be uncovered in this chapter.

\footnotetext{
${ }^{3}$ JPLH website: http://lco.jplh.or.id

${ }^{4}$ PULSE website: http://www.pulse.web.id
} 


\section{Related Works}

In 1996, the National Digital Library of Theses and Dissertations (NDLTD) was launched by the Virginia Tech project team to improve graduate education in US. Due to wider international interest, the NDLTD was renamed in 1997 as the Networked Digital Library of Theses and Dissertations (Fox, 1999). When we established the IndonesiaDLN in 2000, the NDLTD already had more than 70 members across countries. They published and shared their collections within the network. This shows that developing a DL is not merely about developing an independent system moreover it is a part of a global information infrastructure.

Many discussions and efforts have been made to develop a DLs framework that can lead to specification of protocols when various components are involved and many DL servers are integrated (Fox, 1999b). For example, Powell (1998) developed a scalable system for searching heterogeneous multilingual collections on the World Wide Web and the first production was the NDLTD Federated Search. Other initiative that produced a protocol for metadata harvesting over various repository configurations is the Open Archive Initiative (OAI). The OAI Protocol for Metadata Harvesting (OAI-PMH) provides an application-independent interoperability framework based on a metadata harvesting mechanism (OAI, 2003). The protocol has been developed since 1999 and the latest version, protocol version 2, has been released in February 2003. The numbers of institutions that adopt the protocol are increasing by years.

Since the OAI-PMH focuses on the harvesting metadata from a data provider, it requires the data provider to be connected to the Internet for 24 hours a day. If the IndonesiaDLN adopted the protocol without any modification, most of its members will not be able to join the network. More than $50 \%$ of them are connected to the Internet using dial up connections or located behind proxy servers. Thus other members won't be able to harvest their metadata due to the unpredicted availability of the servers.

One year before IndonesiaDLN was established in 2000, there had been a virtual library network initiative, which was called the Indonesian Christian University Virtual Library $\left(\right.$ InCU-VL ${ }^{5}$ ). This network was originally for the Christian universities community, which was pioneered by the Petra Christian University in Surabaya. Then in 2003, it was known as SPEKTRA Virtual Library $\left(\right.$ SVL $\left.^{6}\right)$ after it broadened the scope of its members.

Institutions can join the SVL by buying and using the New SPEKTRA proprietary software. Nowadays, there is no automatic mechanism for the institution's SVL server to synchronize its metadata to other SVL servers. The process of creating union database is by uploading database record through online web interface manually.

\section{Concept and Design}

\footnotetext{
${ }^{5}$ InCU-VL website: http://incuvl.petra.ac.id

${ }^{6}$ SVL website: http://svl.petra.ac.id
} 
Societies or communities that become targets of the IndonesiaDLN are individuals and institutions that want to share and get knowledge through the network. They are not limited only from the academic and research communities, but any parties that want to join the knowledge sharing initiative can become member. Currently, communities that have expressed their interest to join the network are schools, universities, research institutions, NGOs, governmental institutions, public organization, Small and Medium scale Enterprise (SME), etc.

Their knowledge domains are heterogeneous, including children education, heritage, agriculture, health, theses and dissertations, environment, etc. Thus, the digital resources also vary including texts, audios, video, images, source codes, etc. As long as the resources were created, owned, or the copyright allows these resources to be disseminated publicly, then IndonesiaDLN will accept the resources.

\section{The Network Model}

The IndonesiaDLN network model was designed to motivate and enforce communities to join the knowledge sharing initiative. The concept was based on a psychological aspect of their communication nature. Usually, people from the same community will have frequent interaction among them rather than with people from other communities. Instead of inviting them to join into one big network, we suggest them to develop their own networks that consist of their community members. They can be grouped together into a cluster network according to their knowledge domains or regions. For example, there will be networks of Agriculture, Health, Children, East Java Universities, and so on. IndonesiaDLN will act as the bridge for these networks.

Each cluster will manage its own activities, including registration of new nodes, managing users, maintaining cluster hub server, socialization of the knowledge sharing initiative, and provide services to its users. By bringing these administrations to the cluster level, in turn it will decrease the workload at the central network of the IndonesiaDLN.

The IndonesiaDLN finally will consist of networks of digital libraries, or become a network of networks, as shown by Figure 2. In this example, IndonesiaDLN consists of a central hub server and nine cluster networks including children, human rights, heritage, Electronic Theses and Dissertation (ETD), school, religion, environment, health, and agriculture. The central hub server acts as a bridge for the knowledge exchange among the cluster networks or knowledge islands.

The Figure also shows the example of an ETD cluster network. This cluster consists of nine university nodes, four individual nodes, and an Internet café. It has one cluster hub server that bridges their resource exchanges. 


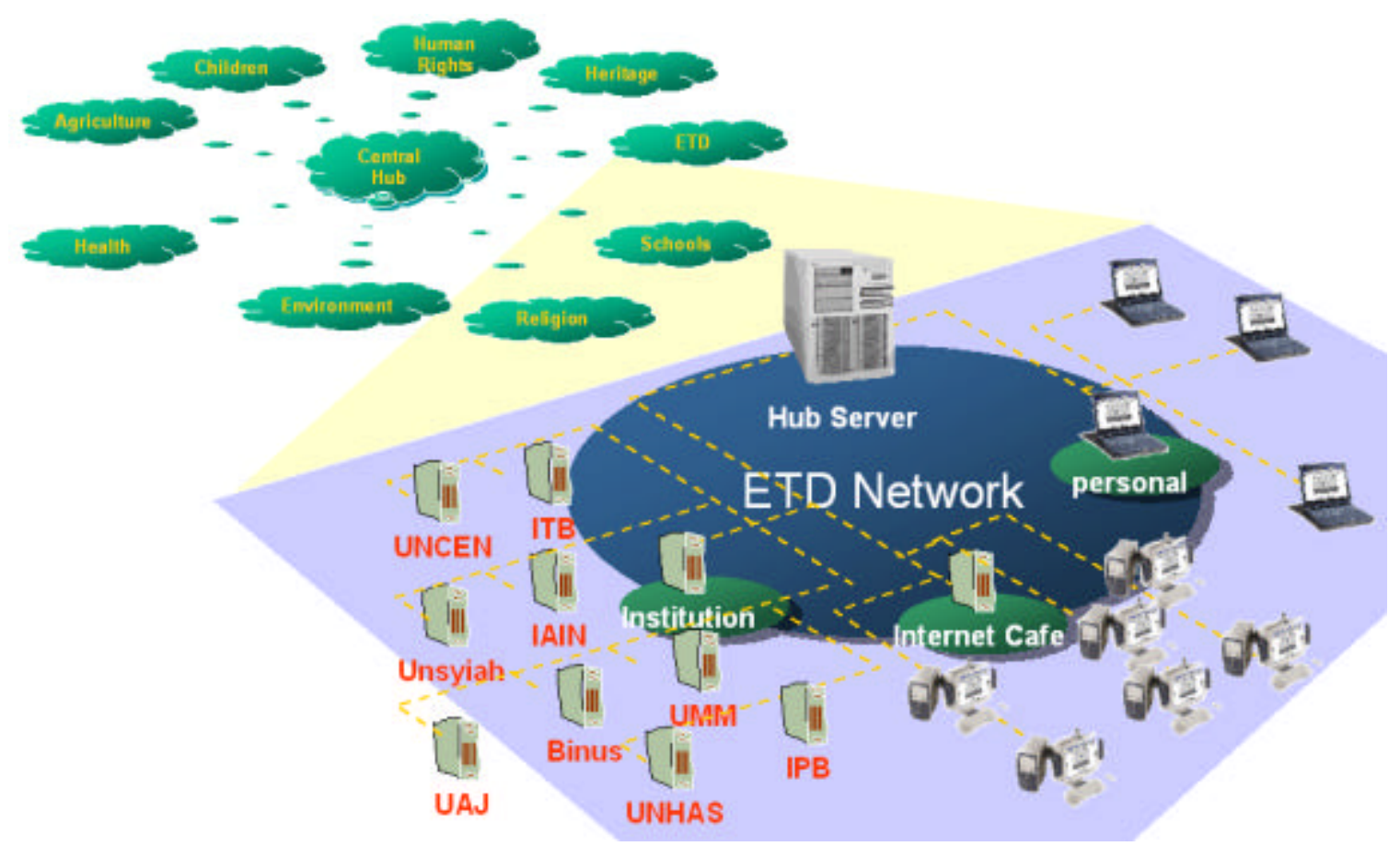

Figure 2. The IndonesiaDLN bridges the networks of digital libraries. This model is called the Network of Networks.

\section{How It Works?}

This section describes in more detail how the networking model of the IndonesiaDLN system above works.

\section{Metadata and Resources}

A DL system manages digital resources such as documents, images, audios, videos, or source codes. Every resource consists of one or more files that are represented by one metadata. In practice, metadata is defined as a structured set of descriptive elements to describe an information resource or any definable entity.

We use the Dublin Core metadata specification to describe the digital resources. This specification contains 15 element terms, i.e. Title, Creator, Subject, Description, Publisher, Contributor, Date, Type, Format, Identifier, Source, Language, Relation, Coverage, and Rights (DCMI, 2003). In our implementation, we add several subelements to accommodate more detailed information about the resource. 


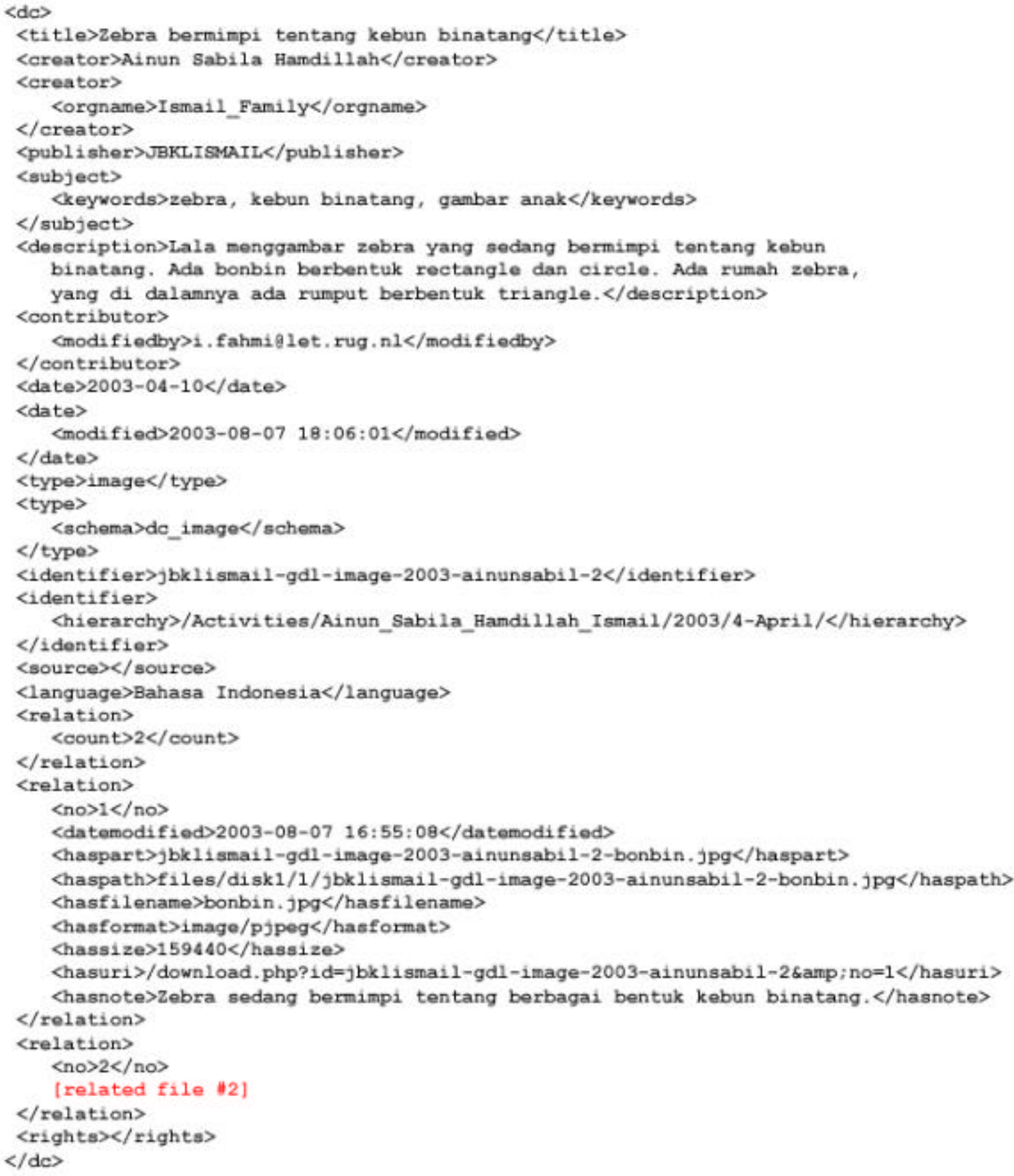

Figure 3. A metadata in XML format provides short description about a digital resource and links to its related files.

We use XML format to encode the metadata as shown by Figure 3. This metadata example describes a children's drawing (image) entitled Zebra bermimpi tentang kebun binatang 'A zebra is dreaming about a zoo' (title) that contains two image files (relation). The metadata also provides other information such as creator, publisher's identifier, subject, description, contributor's email, date, metadata's identifier, source, language, and rights. 


\section{Identifier}

The example of metadata in Figure 3 also describes important information about identifiers. There are two identifiers, publisher's identifier (e.g. JBKLISMAIL) and metadata's identifier (e.g. jbklismail-gdl-image-2003-ainunsabil-2).

Every node (publisher) in the IndonesiaDLN should have a unique identifier, which is required when it exchanges metadata with other nodes. We follow the existing library code convention that is suggested by the National Library of Indonesia to identify every library using a unique code. The code contains information about the library's location, type of its institution, and its institution name. For example JBPTITB is the code for ITB Central Library, where JB stands for Jawa Barat 'West Java' (location), PT for Perguruan Tinggi 'University' (type), and ITB for Institute of Technology Bandung (name). Thus, JBKLISMAIL in the metadata example stands for Jawa Barat (location) Keluarga 'Family' (type), and Ismail (institution or individual name).

The construction of a metadata identifier, for example in jbklismail-gdl-image-2003ainunsabil-2, also uses the publisher's identifier (source). The metadata identifier contains information about its sources (e.g. jbklismail), digital library software name (e.g. $g d l$ 'Ganesha Digital Library'), type of the resource (e.g. image), year of creation (e.g. 2003), creator's name (e.g. ainunsabil), and the serial number of its creation (e.g. 2). With this naming model, it would be fast and easy to grab important information of a metadata only from its identifier.

\section{The Implementation of the OAI Requests and Responses Protocol}

We only disseminate metadata which size is much smaller that the original resource's file size, while the resource files will remain at the original server. If the original server is not connected in 24/7, administrator can upload the files into its hub server.

Figure 4 shows how the metadata and files are stored and exchanged. This Figure illustrates an example of a cluster network in the IndonesiaDLN. It consists of a hub server and three node servers (A, B, and C). The nodes may use permanent (B) or temporary $(\mathrm{A}$ and $\mathrm{C})$ connection to the Internet. 


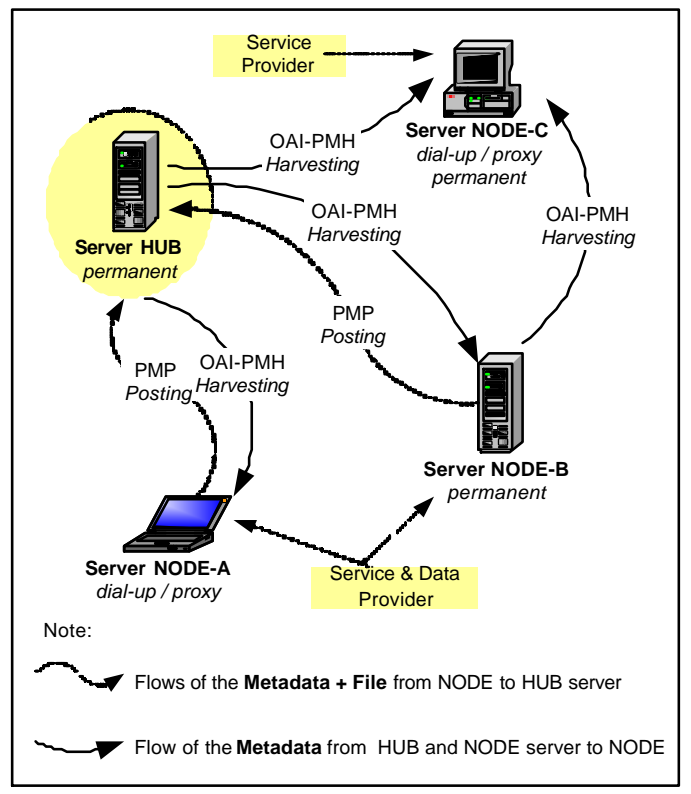

Figure 4. The IndonesiaDLN uses both protocols for metadata posting and harvesting to disseminate the metadata.

The metadata exchange process is started when a data provider (A or B) stores a digital resource containing a metadata and file(s). It posts the metadata into a hub server, using the Protocol for Metadata Posting (OAI-PMP), to build a union metadata collection. This protocol is developed for the IndonesiaDLN by adapting the Open Archive Initiative (OAI-PMH) protocol model.

OAI is an international effort to develop a standard for metadata harvesting protocol among digital library servers (OAI, 2003). The protocol is very simple which is based on requests and responses services on top of the HTTP protocol. When we developed the IndonesiaDLN, the metadata posting services was not supported by the OAI-PMH. For this reason, we had to develop these services (OAI-PMP) for our implementation.

All nodes are allowed to make a mirror of the collection into their local repositories by using the Protocol for Metadata Harvesting (OAI-PMH). With these repositories, the nodes can provide metadata searching and browsing services to their users locally. Whenever the users need to download the files, they will be redirected to the location of the files seamlessly. 


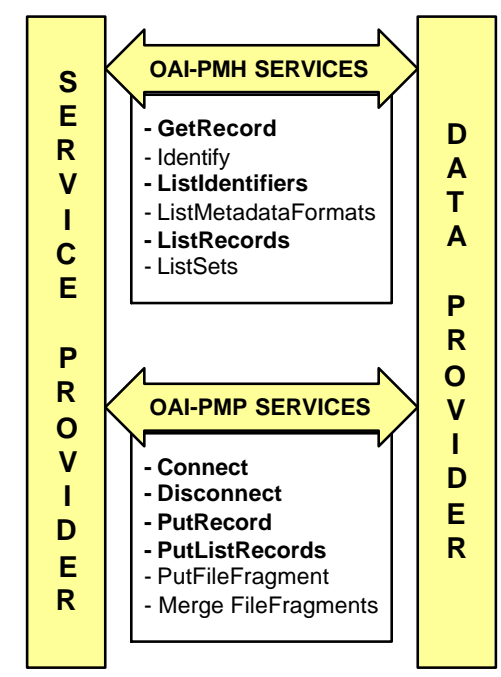

Figure 5. The IndonesiaDLN uses the OAI-PMH services, which extends it with additional services (OAI-PMP) to make possible institutions without 24/7 Internet services to join the network.

Figure 5 shows the diagram of the OAI-PMH and OAI-PMP services in the IndonesiaDLN. The services link data provider and service provider or between publisher nodes. Basically, OAI-PMH provides five request verbs i.e. GetRecord, Identify, ListIdentifiers, ListMetadataFormats, ListRecords, and Listsets (OAI, 2003). For our implementation, we add six request verbs (OAI-PMP) i.e. Connect, Disconnect, PutRecord, PutListRecords, PutFileFragment, and MergeFileFragments (Fahmi, 2002).

The first two verbs of the OAI-PMP, Connect and Disconnect, are for server authentication purpose before it is allowed to post or harvest metadata. The server uses PutRecord and PutListRecords verbs for metadata posting, whether one or many metadata in one posting session. The last two verbs, PutFileFragment and MergeFileFragments are used for file posting purpose.

We use the HTTP POST method with content-type: application/x-wwwform-urlencoded for PutRecord and PutListRecords. In practice, the metadata posting by digital library administrator is carried out through a web-based interface as shown by Figure 6 . The screenshot gives the illustration of a metadata posting using the Ganesha Digital Library (GDL) software. The right box tells us that 12 metadata records have been posted to the 'agri' hub server. The list of the metadata identifiers is also shown under the progress bar. 


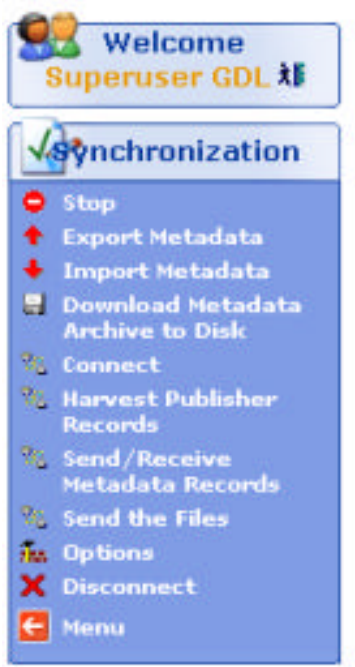

\section{Posting Metadata to 'agri' \\ Posting Metadata $1-12$ of $12 \ldots$}

Successfully Posting Metadata: 12 meconds, size 29444 bytes.

Metadata ID(s): jbklismail-gdl-image-2003-ainunsabil-1(*); jbklismail-

gdl-image-2003-ainuns abil-2 (*), jbklismail-gdl-image-2002-ainunsabil$3(*)$; jbklismail-gdl-image-2002-ainuns abil-4 $\left(^{*}\right)$; jbklismail-gdl-image2003-ainuns abil-5 (*); jbklismail-gdl-image-2003-ainunsabil-6 (*);

jbklismail-gd-image-2003-ainunsabil-7 (*): jbklismail-gdl-image-2003ainulmalik-8 (*)] jbklismail-gdl-image-2003-ainulmalik-9 (*)!

jbklismail-gdl-image-2003-ainunsabil-10 (*)j jbklismail-gdl-image-

2002-ainunsabil-11 (*); jbklismail-gdl-image-2003-ainulmalik-12 (*);

Error message:

Warning: Number of records received (12) by hub server doesn't match

with countRecords ( 30$)$.

Figure 6. The screenshot of the metadata posting process using GDL software. The left box contains the synchronization menu, and the right box shows the progress of the posting.

The detailed request of PutListRecords verb and its response, which is displayed by above screenshot, can be found in Figure 7. This verb requires three arguments, i.e. PHPSESSID, countRecords, and resumptionToken. In this example, the node is sending maximum 30 metadata records into a hub server, which is named agri. Each record contains information about its metadata header (identifier, status, datestamp) and metadata content (see Figure 4). 


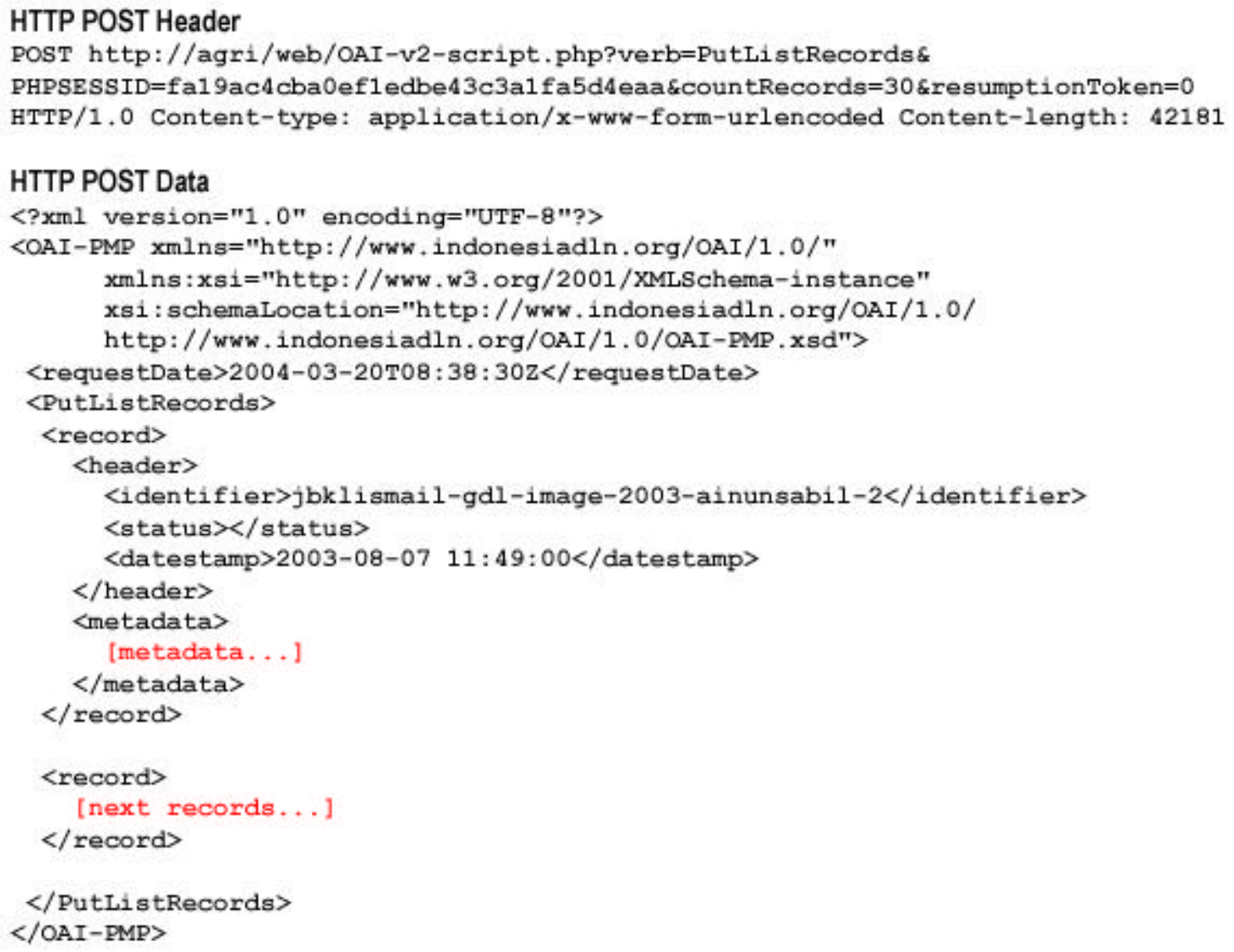

Figure 7. An Example of a request and response strings for the Put List Records verb, which progress is shown by figure 6.

For the PutFileFragment verb, we use the HTTP POST method with contenttype: multipart/form-data. Web browser to post file(s) using HTTP protocol also uses this content-type. A file is sent fragment-by-fragment to a hub server which size can be adjusted according to the Internet bandwidth of the server. Screenshots in Figure 8 show the example of this process.

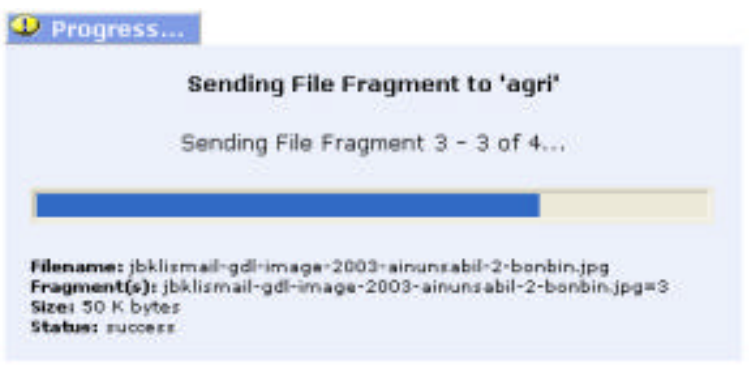

(a)

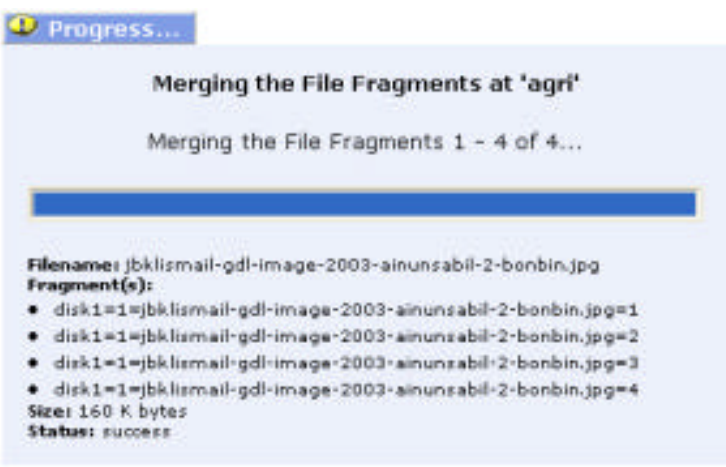

(b) 
Figure 8. Screenshots of a file posting process. (a) A file entitled jbklismail-gdl-image2003-ainunsabil-2-bonbin.jpg is sliced into four fragments in 50 Kbytes each. The fragment is sent one-by-one using the PutF i leFragment verb. (b) The hub server reconstructs the original file by merging all of the file fragments, after receiving MergeFileFragments request from the node.

\section{Hierarchical Category Presentation Model}

The hub server will contain metadata from various digital library nodes. The metadata collection should be organized and presented in a certain way so that it could boost the eagerness of the institution to keep sharing their knowledge. One solution is through the presentation of the metadata collections based on their cluster network and institution name. Thus, the network domains, locations of the source, subject diversities, and richness of the collections (number of metadata records) can be seen from a hierarchical category tree as shown by Figure 9. The top level is the IndonesiaDLN category, then followed by cluster network, institution names, and local categories of the institution (Figure 9b).

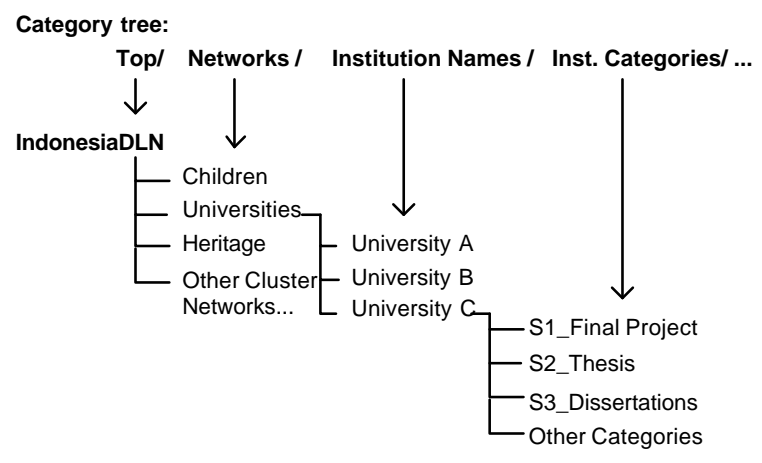

(a)

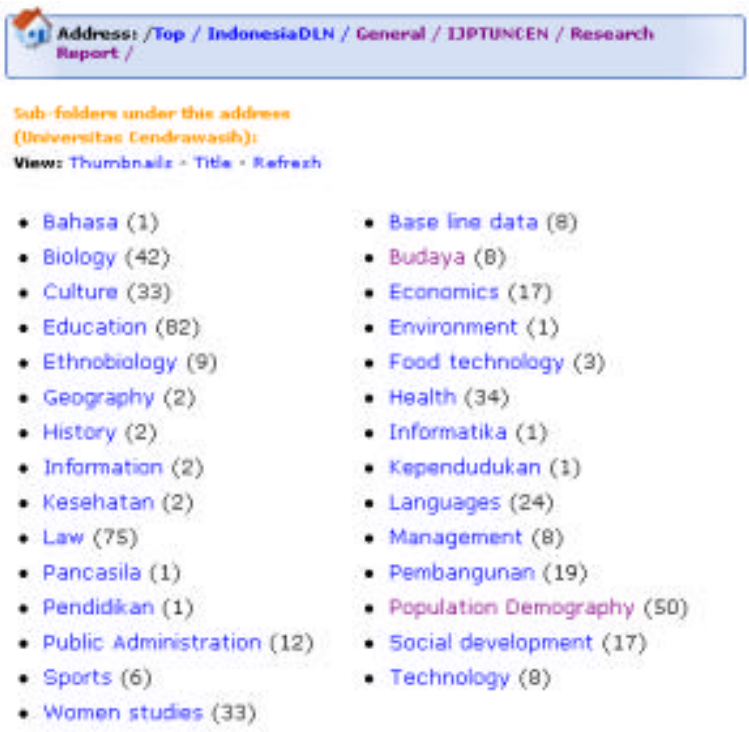

(b)

Figure 9. Information resources in the IndonesiaDLN are presented in a hierarchical category tree. (a) Category tree model of the IndonesiaDLN. (b) An example of the local category tree of University of Cendrawasih (IJPTUNCEN).

The hierarchy sub-element of an IndonesiaDLN metadata provides information about an institution's local category. For example, in Figure 3, the category tree of the metadata is /Activities/Ainun_Sabila_Hamdillah_Ismail/2003/4-April/. With this information in the metadata, all nodes can reconstruct category trees as originally created by the source nodes. 


\section{Implementation}

In this section, we present statistics of the implementation results, which was processed from the access log of the central hub and the node server of ITB Central Library. The data was collected since the launching of IndonesiaDLN at 2001/5/17 until 2003/6/13.

\section{The Open Source Software}

The first software that was developed to implement the previously described concept is the Ganesha Digital Library (GDL). This software is distributed as open-sources and can be downloaded for free from http://gdl.itb.ac.id (GDL, 2003). It is written in PHP scripting language, using MySQL database, and incorporating the SWISH-E (Simple Web Indexing for Humans - Enhanced ${ }^{7}$ ) for indexing and searching the metadata.

Figure 10 shows the screenshot of the GDL interface, which was installed by anonymous institution, ACME Digital Library. The page displays the list of DL nodes in the IndonesiaDLN, which metadata has been harvested by this institution. Each item contains information about Publisher ID, institution name, number of shared metadata, and links to the deeper categories.

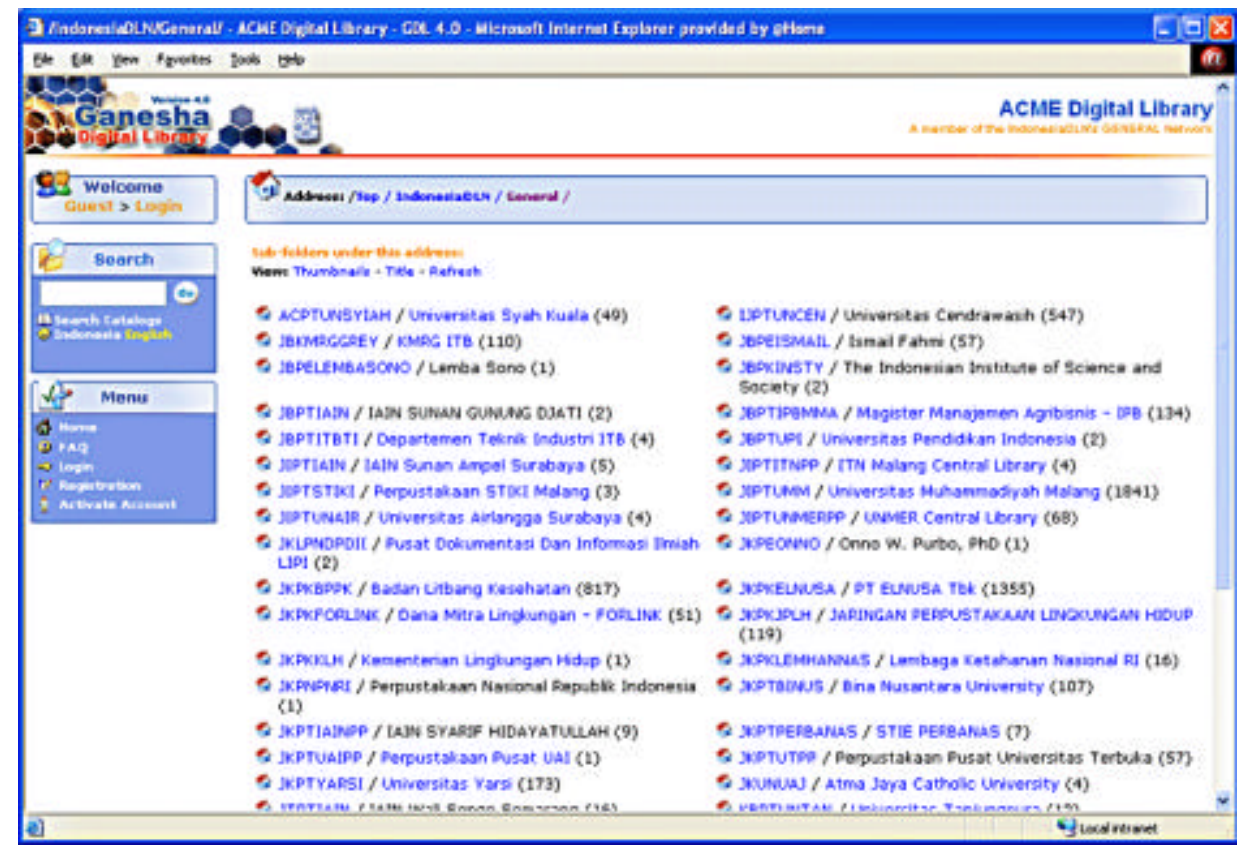

Figure 10. A Screenshot of the GDL shows the list of digital library nodes and number of shared metadata.

User can read the detailed metadata and download the related files through an interface as shown by Figure 11. The download links will bring user to the source server that stores the files. An authentication can be set so that only members that get the download access.

\footnotetext{
${ }^{7}$ SWISH-E website: http://swish-e.org
} 


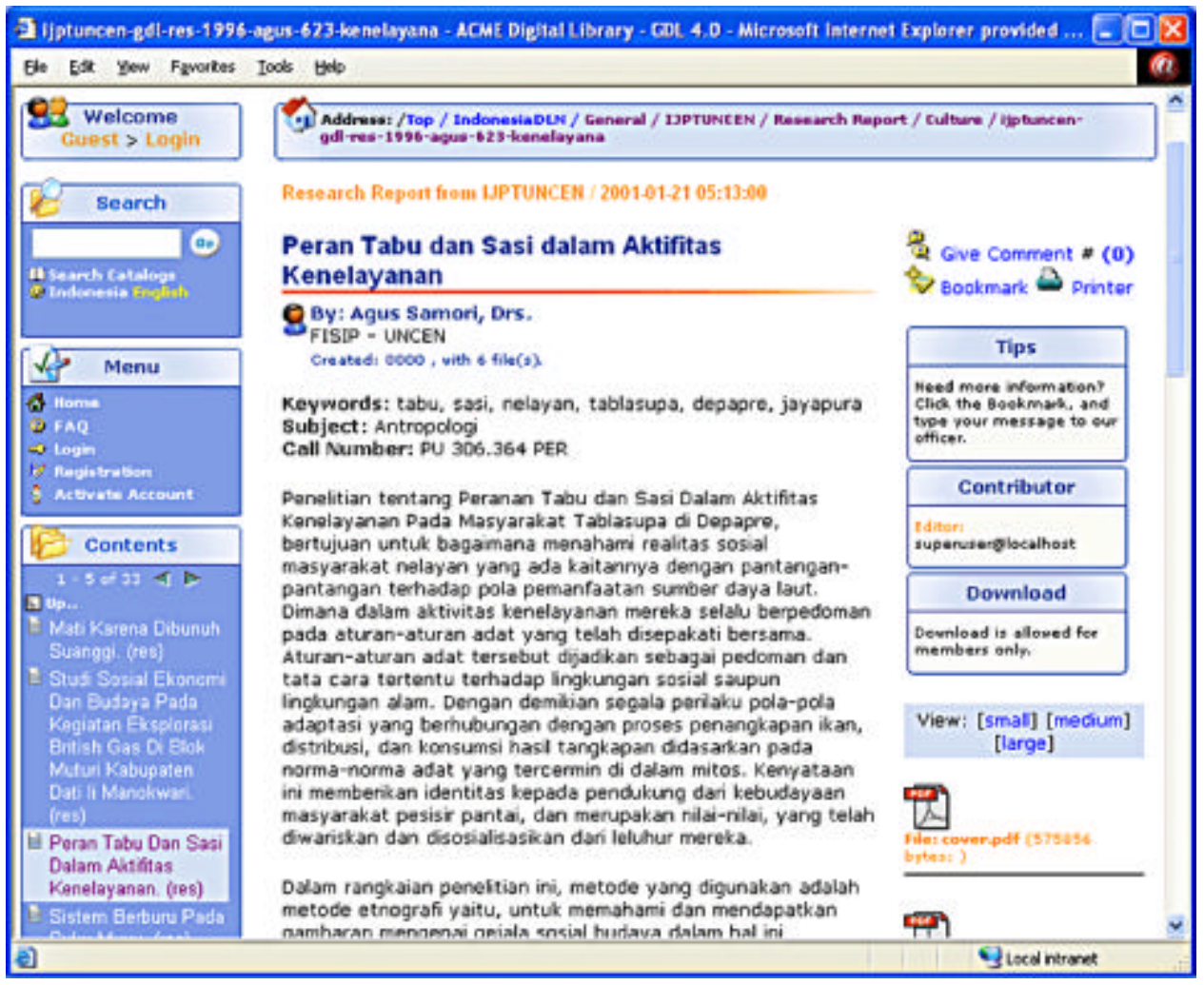

Figure 11. The screenshot of GDL provides content of metadata and links to the related files.

\section{Profiles and Activities of the Nodes}

Currently, there are 94 nodes registered at the central hub. 50 nodes use dedicated connection, and 44 nodes use dial up or temporary connection. From these numbers, there are 48 nodes that have shared their collections, and 59 nodes that have harvested collections from central hub.

Figure 12 shows the skewed distribution of the number of metadata and files shared by the nodes. There are 5 nodes that share more than 500 metadata, 12 nodes share more than 100 metadata, and 22 nodes share more than 30 metadata. The top five nodes are University of Muhammadiyah Malang, Institut of Technology Bandung, Elnusa, Indonesian Health Research, and University of Cendrawasih. The last mentioned node is located at the eastern most of Indonesia and uses dial-up connection. 


\begin{tabular}{|c|c|c|c|}
\hline No & Node D & Eoladata & Fine \\
\hline & JIPTUMM & 1841 & 4019 \\
\hline$\overline{2}$ & JBPTITBPP & 1501 & $\overline{1280}$ \\
\hline 3 & MPKENNSA & 1305 & 7 \\
\hline 4 & NKPKBPPK & 817 & 85 \\
\hline $\mathbb{E}$ & ICPTUNCEN & 847 & 13 \\
\hline 6 & SAPTUNERAT & 300 & 8 \\
\hline & |SPTYAR8| & 173 & 167 \\
\hline 8 & LAPTIAIN & 136 & 102 \\
\hline 8 & LBPTIPBMMA & 134 & 138 \\
\hline 10 & KKPKJPLH & 119 & 0 \\
\hline 11 & JEKMROGREY & 710 & 5 \\
\hline 12 & KPTBINUS & 107 & 42 \\
\hline 13 & JIPTUNNERPP & 68 & 0 \\
\hline 14 & SRPTUTPP & Sy & 48 \\
\hline 16 & RBPEISMAL & $\overline{57}$ & BE \\
\hline 16 & JKPKFORLINK & 51 & 27 \\
\hline 17 & ACPTUNSYIAH & 49 & 48 \\
\hline 18 & KSPTIAIN & 46 & 16 \\
\hline 19 & CDLHUB & 40 & 0 \\
\hline 20 & SNPTIANN & 36 & 24 \\
\hline
\end{tabular}

\begin{tabular}{|c|c|c|c|}
\hline No & Node D & Entaden & Files \\
\hline 21 & SSPTLAN & 35 & 4 \\
\hline 22 & SUPTIAIN & 30 & 22 \\
\hline 23 & JTPTIAIN & 16 & 6 \\
\hline 24 & JKPKLENHANNA & 16 & 63 \\
\hline 28 & SBPTRIN & 10 & 4 \\
\hline 29 & KBPTUNTAN & 12 & $\overline{1}$ \\
\hline 27 & DKPTIANPP & $\theta$ & 5 \\
\hline 28 & RIPTIAIN & 7 & 0 \\
\hline 29 & JKPTPERBANAS & 7 & 6 \\
\hline 30 & JIPTIAN & 5 & 2 \\
\hline उ1 & JIPTUNAR & 4 & 0 \\
\hline 32 & JKUNUAN & 4 & 3 \\
\hline 33 & JBPTITBTI & 4 & 3 \\
\hline 34 & JIPTITNPP & 4 & 4 \\
\hline 36 & TESTINSTITUTIO & 4 & 2 \\
\hline 36 & JIPTSTIKI & 3 & 1 \\
\hline$\overline{31}$ & SCPTUNHALU & 3 & 4 \\
\hline 38 & DEPTIAN & 2 & D \\
\hline 39 & JBPKINSTY & 2 & 2 \\
\hline 40 & JKLPNDPD|| & 2 & 1 \\
\hline
\end{tabular}

Figure 12. Number of metadata and files shared by the IndonesiaDLN nodes.

The number of attempts to harvest metadata by the nodes is also skewed, as shown by Figure 13. The vertical axis describes number of attempts to harvest metadata from the central hub. There are 12 nodes that have attempted to harvest metadata more than 5000 times, 23 nodes more than 1000 times, and 41 nodes more than 100 times.

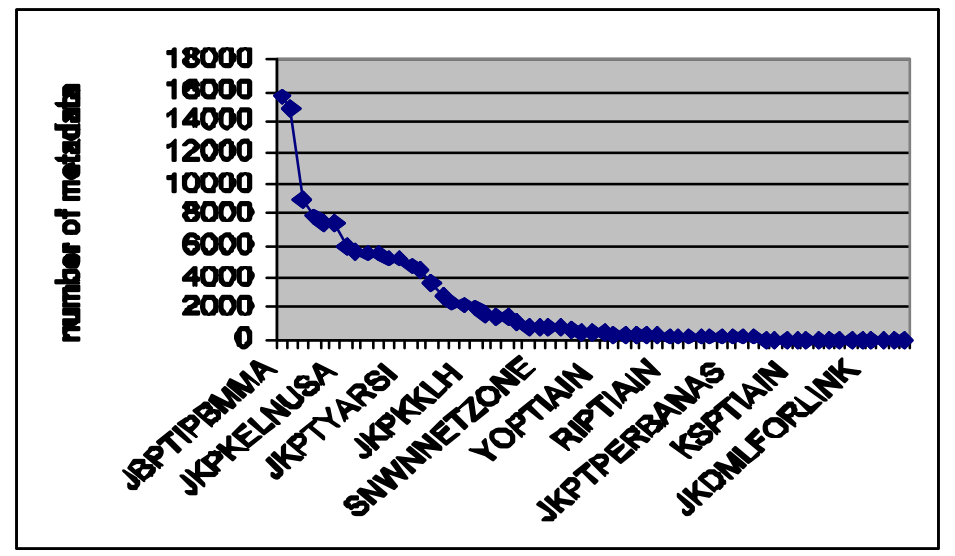

Figure 13. The distribution of the total number of metadata harvested by each of 59 nodes.

Figure 14 (a) shows the access trend of each node to the central hub, while Figure 14 (b) shows their cumulative access. From these figures we know that the cumulative access tends to increase as the number of collection also ncreased. But only few nodes that contribute to this increase, especially by new nodes and some most active nodes. There are many nodes that only active at the beginning of their implementation. Usually, it is caused by the lack of maintenance and operation plan. 


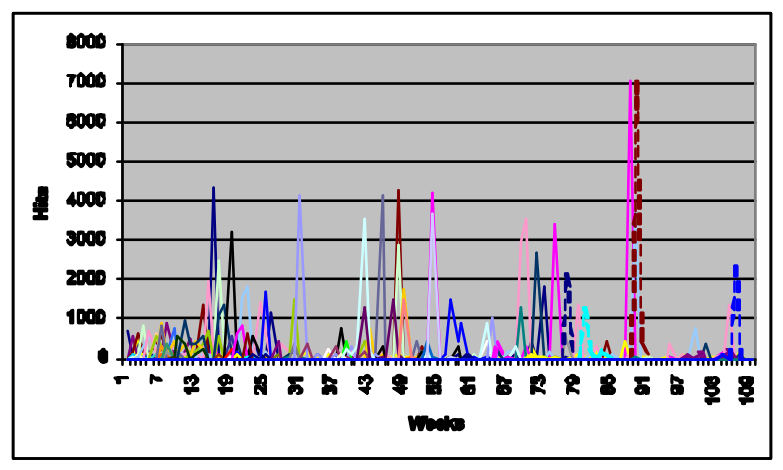

(a)

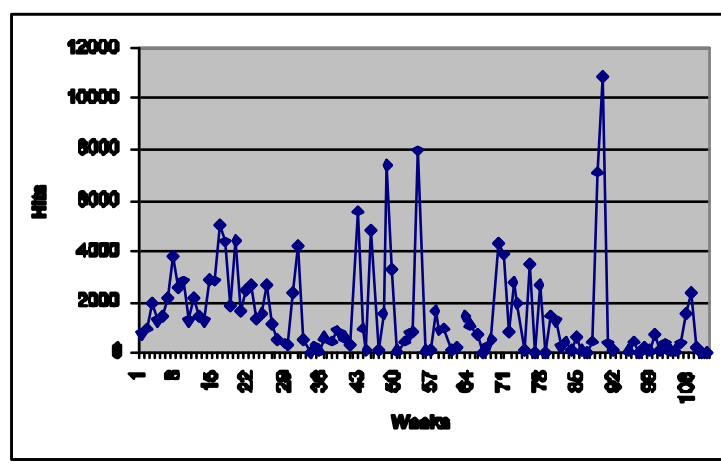

(b)

Figure 14. (a) The trend of nodes' access to the central hub. (b) The cumulative access to the central hub.

These figures indicate that we need to increase efforts for the socialization, maintenance, education, and technical support especially to the existing nodes. While maintaining the whole nodes is a difficult job for the voluntary IndonesiaDLN, we expect that the realization of the cluster networks will able to solve this problem. Each network will manage these efforts for its existing nodes.

\section{Users Profile}

Most of our users are from Indonesia (98\%). Another countries that contribute 10 to 20 users are Malaysia, Japan, Australia, Singapore, Germany, USA, UK, Canada, and India. They come from all big islands of Indonesia such as mostly from cities in Java island (e.g. Bandung, Jakarta, Malang, Yogyakarta, Surabaya, Bogor, Semarang, etc), Sumatera (e.g. Medan, Palembang, Padang, Aceh, etc), Sulawesi (e.g. Makassar, Manado), Kalimantan (e.g. Pontianak, Banjarmasin, etc), Irian Jaya (e.g. Jayapura), etc.

There are ten universities that contribute more than 50 users as shown by figure 15 (a). Other users are from hundreds of education institutions, private companies, organizations, and individuals.

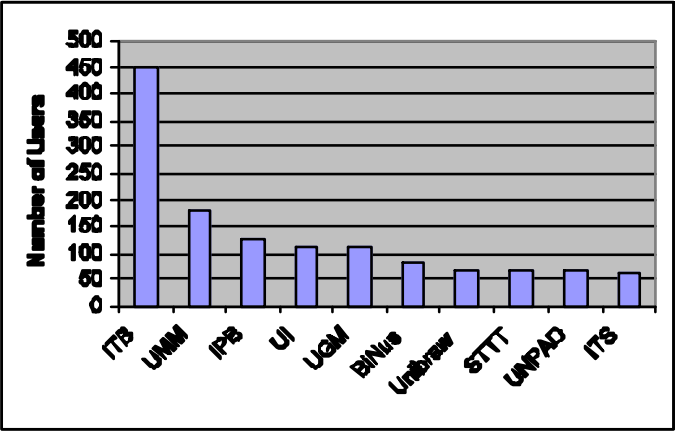

(a)

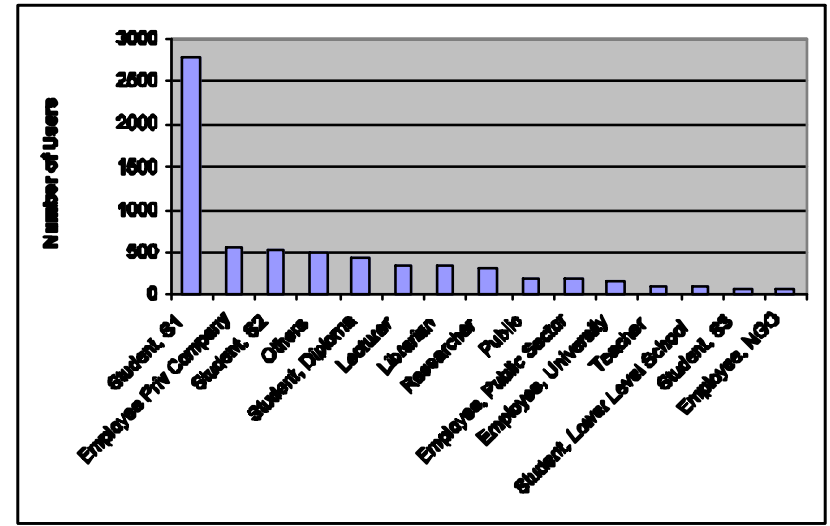

(b) 
Figure 15. (a) Top 10 users' institutions. (b) The profile of users' background.

Most of them are undergraduate students as shown by figure 15 (b). What interesting from the last figure is that users from private company are in the second position. Possibly this shows the need for a kind of long life learning, or they need resources from the universities and research institutions to support their works. This information should become a motivation for any institutions to provided more resources in their digital library and more important to provide services to these users.

There are more than 100 teachers and 90 students from schools that become our users. The actual number must be higher, because only the central hub captured this data. This shows that the school communities also interested to get benefits from these kinds of information sources. This information will lead us to the initiatives of developing digital library network for schools, from the kindergarten to the high schools.

Users can register at any nodes. Once they are registered, their accounts can be used to access all other nodes as long as the nodes where they are registered have synchronized or posted the data to the central hub. Figure 16 shows the number of users according to the nodes where they registered. The distribution is also skewed. Most of them are registered at JBPTITBPP (node at ITB Central Library). It doesn't mean that all of them are from ITB because anybody from any institution can register at any nodes.

\begin{tabular}{|c|c|c|}
\hline No & Node ID & Werber \\
\hline & JAPTITEPP & 4922 \\
\hline & GDLHUB & 797 \\
\hline 3 & JKPKBPPK & 269 \\
\hline 4 & JAPTIPEMMA & 181 \\
\hline 5 & JKPTBINUS & 161 \\
\hline 6 & JIPTUMM & 67 \\
\hline 7 & JKPKELNUSA & 51 \\
\hline 8 & ACPTUNEYIAH & 51 \\
\hline 9 & BAPTUNSRAT & 36 \\
\hline 10 & TESTNSTITUTION & 33 \\
\hline 11 & JKPTYARSI & 28 \\
\hline 12 & SGPTUNHALU & 20 \\
\hline 13 & SNWNNETZONE & 18 \\
\hline 14 & JIPTLIN & 17 \\
\hline 15 & JAPTUPI & 17 \\
\hline 16 & JKPKWPLH & 11 \\
\hline 17 & RSPTIAN & 10 \\
\hline 18 & JKPTIAINPP & 10 \\
\hline 19 & JKPTPERBANAS & 10 \\
\hline 20 & BBPTIAN & 10 \\
\hline 21 & RIPTIAIN & 1 \\
\hline
\end{tabular}

\begin{tabular}{|c|c|c|}
\hline No & \begin{tabular}{|l} 
Node ID \\
\end{tabular} & Dember \\
\hline 22 & SUPTIAN & 10 \\
\hline 23 & JKPKKLH & 8 \\
\hline$\overline{24}$ & ENPTIAN & \\
\hline 25 & JPTUNMERPP & \\
\hline 28 & LPTUNCEN & \\
\hline 27 & JKUNUAJ & \\
\hline 28 & JIYPIA & \\
\hline 28 & JPTSTIKOMSAY & \\
\hline 30 & JPTUNAIR & \\
\hline 31 & JKLPNDPD|| & \\
\hline 32 & JIPTSTIK| & \\
\hline 33 & JBPTITEE & \\
\hline 34 & JUNUMM & \\
\hline 35 & JPTITNPP & \\
\hline 36 & JKDMLFORLNK & \\
\hline 37 & JKRPKFORLINK & \\
\hline 38 & JBPEISMALL & \\
\hline 30 & JBPTITBPSUD & \\
\hline 40 & JKWHNEDL & \\
\hline 41 & ACPETOPCAN & \\
\hline
\end{tabular}

Figure 16. Number of users according to the Node ID where they are registered.

\section{Collections}

Most of the collections shared by our nodes are research reports, undergraduate's final project, articles, and master's thesis. This resources types are typical in the academic and research institutions where our nodes mostly from this community. Figure 17 (a) shows the distribution of the resource types. 


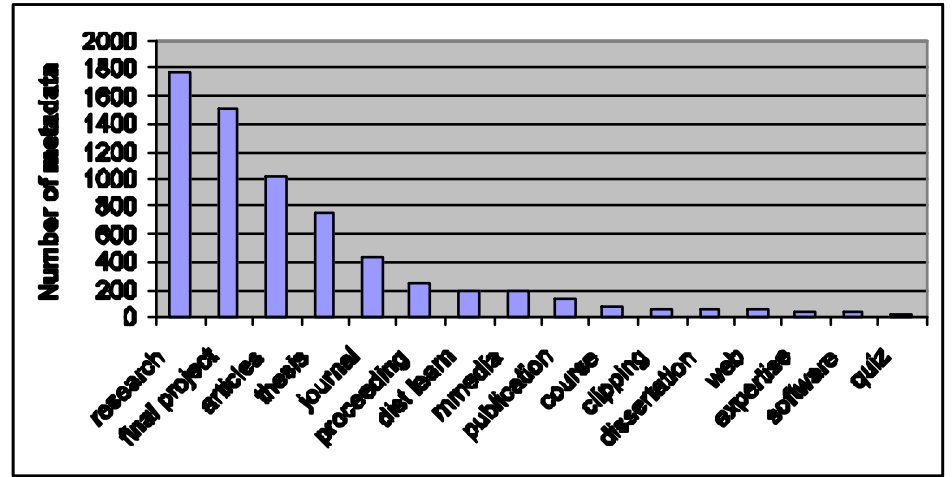

(a)

\begin{tabular}{|c|c|c|}
\hline No & File Formats & Total \\
\hline & UNKNOWN & 4524 \\
\hline & epplieation/pdf & 804 \\
\hline & Imago/pjpeg & 241 \\
\hline 4 & epplication/moword & 138 \\
\hline & epplieution/octat-etream & 122 \\
\hline & toxt/richtext & 102 \\
\hline & audio/mper & 102 \\
\hline 8 & epplieation/Vnd_m-realmedia & 54 \\
\hline 9. & application/vnd_mo-powerpoint & 53 \\
\hline 10. & application/x-zip-comproseod & 52 \\
\hline & audio/x-pn-roalaudio & 20 \\
\hline 12. & texththml & 17 \\
\hline & toxt/plain & 12 \\
\hline 14 & Image/pif & 8 \\
\hline 15: & application/x-mepowerpoint & 7 \\
\hline & andio/mid & 2 \\
\hline & eppollication/vnd_mi-excel & 2 \\
\hline
\end{tabular}

(b)

Figure 17. (a) The types of collection. (b) Format of the associated files.

We encouraged people to upload files together with the metadata. The formats of files are varying, from text document, multimedia, and source code as shown by table in Figure 17 (b). Most of the files are using PDF and MS Word format for documents, and jpg for images. The UNKNOWN format is caused by error in the systems when they captured the detailed information of the uploaded files.

\section{Utilization and Behaviors}

This subsection describes what and how users access the collections. We compare the utilization at the central hub and the node. Access log data for the node was taken from the digital library server at ITB Central Library. Currently, this is the most utilized node in IndonesiaDLN.

Figure 18 gives information about this comparison, according to the cumulative and guest accesses by weeks. From this figure we know that users' access to the node is higher compared with their access to the central hub. The fluctuation is also very close to the guest accesses. Most accesses are anonymous. This information gives a positive expectation that services to end-user will likely be distributed to the nodes. They will experience faster and better accesses to their closest nodes. If the nodes frequently synchronize their collections to the central hub, users don't have to visit the central hub because the search and brows sources are the same. 


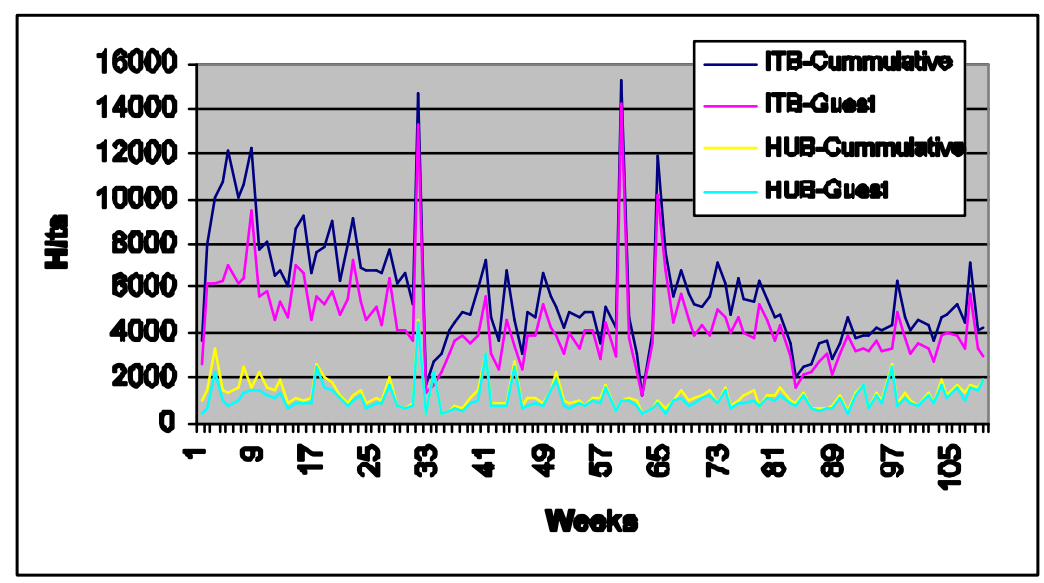

Figure 18. The cumulative and anonymous access to the ITB node and to the central hub.

There are two peaks on the graphic, which were related to the socialization and advertisement of IndonesiaDLN during its second and third annual meeting. The lowest access after the meetings were affected by the new year holidays. During normal weeks, the average accesses are similar. This trend shows that to increase the utilization of collections we need to promote the network intensively. Currently, IndonesiaDLN secretariat hasn't done much effort on this matter. Only before and during the annual meetings we published newsletters, news, and discussion through mailing lists.

\section{Conclusion and Future Works}

The IndonesiaDLN's age is just 2 years since its launching in 2001. The main objective of IndonesiaDLN is to bridge the process barrier and the cultural barrier of the national knowledge process.

We have learned several problems and the most influencing one is the non-technical problem. For this reason, we developed the Network of Networks (NeONs) concept, where every community can develop its own digital library network, manage its knowledge process and sharing, and provide services to its nodes and users. We are in progress of implementing this concept on the ground. Currently, the software that have been improved to accommodate the concept and we are in progress of testing and evaluation.

The realization of the concept is very important because it is based on the user and community behavior, not only based on the technical considerations. This shown has shown that technically, we can give contribution in breaking the barrier of information divide. But, many problems still arise especially on the non-technical matter, such as the administrator motivation, maintenance and operational plan, support from the higher leaders, etc. 
We plan to start the development of the cluster networks especially with partners that have shown their dedication. It is expected that within this year (2003), at least one cluster network can be established.

Currently, more than 90 nodes have been registered at the central hub, and more then 40 nodes have shared their collections. Not all of the nodes keep participating the knowledge sharing. Most of them only active at the beginning.

The networking model using OAI protocol has shown its ability to integrate digital libraries with very limited Internet access where a half of our nodes are using dial-up connection. This problem is common in most developing countries. We expect that this experience can give contribution to us on how to develop a national wide digital library network, especially for the third worlds.

\section{References}

Perpustakaan PT (Perguruan Tinggi) belum Maksimal. (2003, September 24). Media Indonesia. Retrieved March 16, 2004, from http://www.mediaindo.co.id/cetak/berita.asp?id=2003092403523393

Fahmi, I., Utama, M.C., \& Dwiyanto, A.R. 2000. Hasil pertemuan IndonesiaDLN, 3-4 Oktober 2000. Retrieved March 16, 2004, from ????

Fahmi, I., Muharto, R., \& Ibrahim, I.K. 2002. Extending the OAI protocol as the data integration framework for the digital library network in the third world. In Proceeding iiWAS $4^{\text {th }}$ Conference, Bandung, Indonesia, September 12, 2002.

Fox, Edward A., John L. Eaton, Gail McMillan, Neill A. Kipp, Paul Mather, Tim McGoingle, William Schweiker, and Brian Devane. (1997). Networked Digital Library of Theses and Dissertations: An International Effort Unlocking University Resources,' D-Lib Magazine, September 1997. Retrieved October 10, 2003 from the WWW: http://www.dlib.org/dlib/september97/theses/09fox.html.

Fox, Edward A. (1999). FIPSE Final Report: Improving Graduate Education with the National Digital Library of Theses and Dissertations. Retrieved October 10, 2003 from the WWW: http://www.ndltd.org/pubs/FIPSEfr.pdf.

Fox, Edward A. (1999b). Digital Libraries. In R.B. Yates \& B. R. Neto (Eds), Modern Information Retrieval (pp. 415-432). New York: Addison Wesley.

Fox, Edward A., Hussein Suleman, dan Ming Luo. (2002). Building Digital Libraries Made Easy: Toward Open Digital Libraries. ICADL 2002, LNCS 2555, 2002.

OAI. (2003). The Open Archives Initiative Protocol for Metadata Harvesting. February 2003. Retrieved October 16, 2003 from the WWW: http://www.openarchives.org/OAI/openarchivesprotocol.html 
DCMI. (2003). Dublin Core Metadata Initiative (DCMI) Metadata Terms. 19 November 2003. Retrieved March 20, 2004 from the WWW:

http://dublincore.org/documents/dcmi-terms/

\section{Acknowledgement}

IDRC, YLTI 